\title{
Reversible Shape Transition of Pb Islands on $\mathrm{Cu}(111)$
}

\author{
R. van Gastel, ${ }^{1,2}$ N. C. Bartelt, ${ }^{3}$ and G. L. Kellogg ${ }^{1}$ \\ ${ }^{1}$ Sandia National Laboratories, Albuquerque, New Mexico 87185-1415, USA \\ ${ }^{2}$ Solid State Physics, MESA + Institute for Nanotechnology, University of Twente, P.O. Box 217, 7500 AE, Enschede, The Netherlands \\ ${ }^{3}$ Sandia National Laboratories, Livermore, California 94551-0969, USA
}

(Received 13 September 2005; published 26 January 2006)

\begin{abstract}
Using low-energy electron microscopy, we have observed a reversible transition in the shape of $\mathrm{Pb}$ adatom and vacancy islands on $\mathrm{Cu}(111)$. With increasing temperature, circular islands become elongated in one direction. In previous work we have shown that surface stress domain patterns are observed in this system with a characteristic feature size which decreases with increasing temperature. We show that the island shape transition occurs when the ratio of the island size to this characteristic feature size reaches a particular value. The observed critical ratio matches the value expected from stress domains.
\end{abstract}

PACS numbers: 81.16.Dn, 68.35.Md, 68.37.Nq, 68.47.De

A basic requirement to controlling nanoscale surface morphology is an understanding of the factors that affect the arrangement of atomic steps. In particular, understanding what determines the shape of $2 \mathrm{D}$ islands is important. In the absence of long-ranged forces, the equilibrium shapes of 2D islands on surfaces are determined from the anisotropy of step free energies by applying the Wulff construction [1]. If step energies are isotropic, island shapes are circular. However, as has been pointed out by many authors, the Wulff construction can no longer be applied in the presence of the long-range elastic, electrostatic, or magnetic interactions that are present on surfaces [2-8]. As the size of islands increases, these long-ranged forces become more important. In particular, it has been shown that at a characteristic size, determined by a balance between the step energy and the long-ranged interactions, a transition from a compact island shape to an elongated shape should occur to minimize the long-ranged interaction energies. There is experimental evidence that this transition occurs for $\mathrm{Ag}$ islands on $\mathrm{Si}(111)$ and for twodimensional islands of $\mathrm{Si}$ on $\mathrm{Si}(001)$ and $\mathrm{Si}(111)$ [8-10]. For all of these cases the long-ranged force is caused by elastic relaxations due to the surface stress difference between the island and the surrounding terrace. In this Letter we report low-energy electron microscopy (LEEM) [11] observations of a shape transition which occurs for $\mathrm{Pb}$ overlayer islands on $\mathrm{Cu}(111)$. In this system a transition due to surface stress occurs as temperature is varied. This enables us to verify that the transition we observe is reversible. Also, we are able to accurately measure the characteristic size of surface stress domains independent of the droplet transition. We are thus able to verify the predictions of elasticity theory of equilibrium island shapes with increased accuracy.

When submonolayer amounts of $\mathrm{Pb}$ are deposited onto $\mathrm{Cu}(111)$, two phases are observed [12-16]. Below $0.22 \mathrm{ML}, \mathrm{Pb}$ is incorporated into the $\mathrm{Cu}$ surface in the form of a disordered surface alloy. At higher coverages, a condensed $\mathrm{Pb}$ overlayer begins to form. If the deposition temperature is above $650 \mathrm{~K}$, this $\mathrm{Pb}$ overlayer initially occurs as isolated $2 \mathrm{D}$ islands. With increasing coverage these islands are observed to coalesce into well-defined stripes. This stripe phase then evolves into an "antidroplet" phase consisting of circular holes in the $\mathrm{Pb}$ overlayer phase. Increasing the Pb coverage fills the holes. Detailed analysis of these patterns has led to the conclusion that they are surface stress domains $[17,18]$.

Figure 1(a) shows a LEEM image of four antidroplets in a $\mathrm{Pb}$ overlayer phase at a temperature of $570 \mathrm{~K}$. In this image the $\mathrm{Pb}$ overlayer phase appears bright. The antidroplets are the dark circular regions. The dark stripes are regions of the alloy phase decorating atomic steps on the $\mathrm{Cu}$ substrate. The antidroplets were formed by stopping $\mathrm{Pb}$ deposition just short of the saturation coverage of the $\mathrm{Pb}$ overlayer. (For experimental details, see [19].) At this temperature, mass transport is sufficiently slow that the droplets can have diameters considerably different from their equilibrium size [19].

Figure 2(a) shows a LEEM image of the equilibrium striped phase at the same temperature formed at half the saturation coverage. Notice that the diameter of the droplets shown in Fig. 1(a) is comparable to the stripe width. Detailed calculations using a simple isotropic elastic Green's function [20] show that circular droplets becomes unstable with respect to elongation in one direction when the diameter of the droplet is greater than $e^{1 / 3}$ times the equilibrium diameter of an isolated droplet. Because the isolated equilibrium droplet diameter is $e^{2} / 2 \pi$ times the stripe width at half coverage [6], the critical ratio of the droplet diameter to stripe width is $e^{7 / 3} / 2 \pi \approx 1.64$. The droplets shown in Fig. 1 are smaller than this critical size, and hence their circular shape is consistent with theory.

This situation changes, however, when the temperature is increased. As Figs. 2(b) and 2(c) show, the equilibrium stripe width decreases with increasing temperature. (In Ref. [18], this decrease is attributed to a decreasing boundary energy with increasing temperature.) Thus, at these temperatures, the equilibrium stripe width is smaller than 

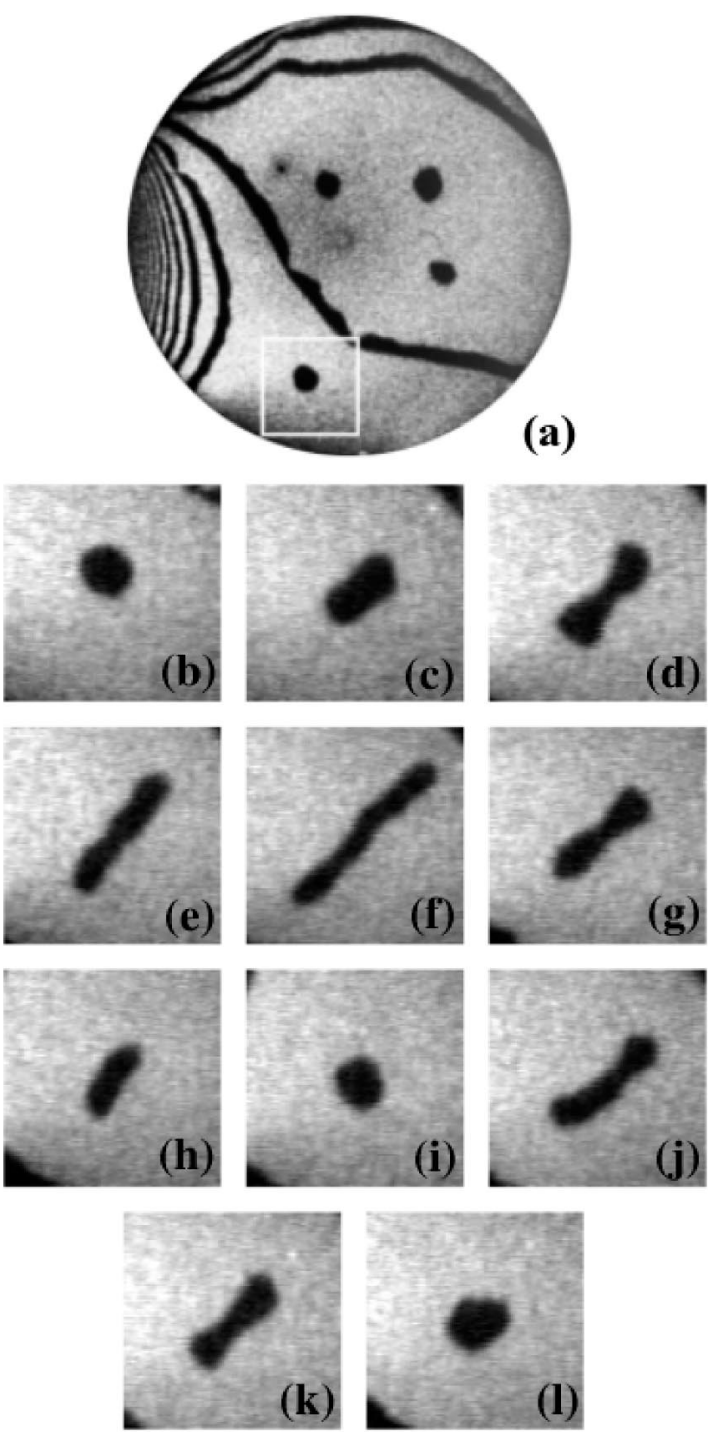

FIG. 1. [(a), $T=570 \mathrm{~K}]$ A $3 \mu \mathrm{m}$ LEEM image of four antidroplets of the surface alloy phase embedded in a matrix of overlayer phase. [(b), $T=573 \mathrm{~K}]$ As temperature is increased, the $0.65 \mu \mathrm{m}$ wide region marked in (a) shows that the initially circular droplet $[(\mathrm{c}), T=593 \mathrm{~K}]$ first assumes an elliptical shape and then [(d)-(f), $T=594-598 \mathrm{~K}]$ becomes further elongated. The reversibility of the transition is demonstrated as temperature is decreased. $[(\mathrm{g})-(\mathrm{h}) T=592-590 \mathrm{~K}]$ The stripe decreases its length and [(i) $T=589 \mathrm{~K}]$ becomes a circular droplet again. Panels (j)-(l) show another cycle of the shape transition of this droplet.

the island diameter shown in Fig. 1(b). One would thus expect that if this island was heated to the temperature of the narrow stripes of Figs. 2(b) and 2(c), maintaining constant area, the island shape would show the influences of long-ranged elastic relaxations. The heating sequence of Figs. 1(b)-1(f) confirms this effect. As the island is heated to a temperature where the island diameter is comparable to the stripe width, there is a sudden change in the island shape-it becomes elongated in one direction. When the

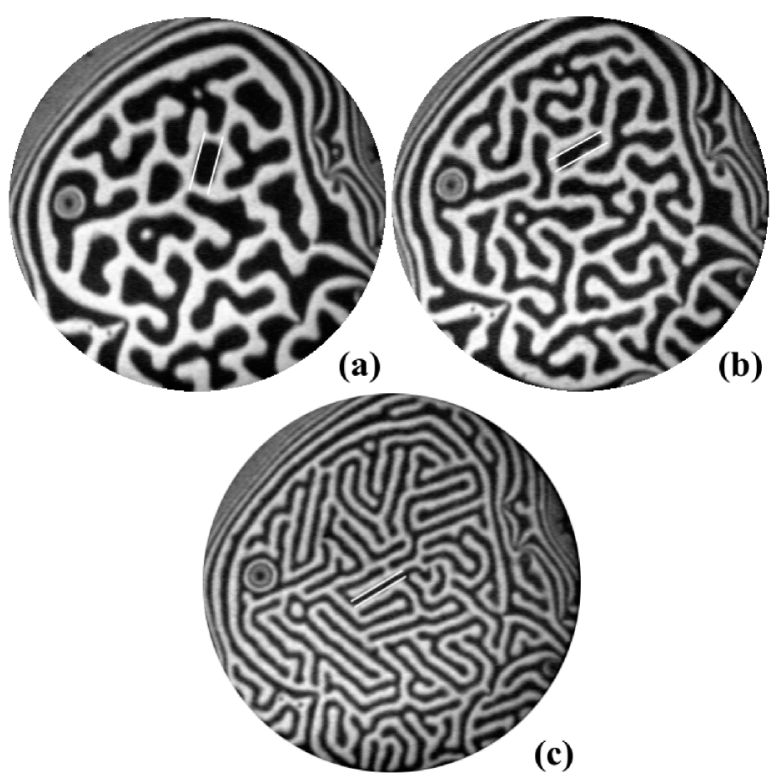

FIG. 2. (a) A $2.85 \mu \mathrm{m}$ diameter LEEM image of a striped phase formed at half coverage at a temperature of $573 \mathrm{~K}$. The average measured stripe width is indicated by the white lines in the center of the image. (b) The same striped pattern at a temperature of $596 \mathrm{~K}$. The increase in temperature has led to a decrease in stripe width. This decrease is also seen at $635 \mathrm{~K}$ in (c).

temperature is lowered, the circular shape is recovered, as shown in Figs. 1(g)-1(i). This shape change can be reproduced precisely - as shown by the subsequent reheating sequence of Figs. 1(j)-1(l). The shape transition was not observed to depend on the heating or cooling rate. This reversibility is consistent with the shape change being an equilibrium effect and not a growth effect, as discussed in Ref. [21]. The direction of the elongation for all of the droplets is always in one of three directions. As argued in Ref. [22], we attribute this to effects of the elastic anisotropy of the substrate.

Figure 3 shows a plot of the aspect ratio as a function of temperature of a sequence similar to the one shown in Fig. 1. The aspect ratio was measured by fitting an elliptical shape to the droplets and measuring the length of the axes parallel and perpendicular to the elongation direction. Below we refer to these axes as "long" and "short," respectively. Also plotted in Fig. 3 is the temperature dependence of the equilibrium island area deduced from the measurements of the stripe width presented in [18]. Clearly the aspect ratio reversibly changes at a well-defined critical temperature when the equilibrium droplet size reaches a particular value. This sudden shape transition (as opposed to a continuous evolution in shape) is what has been predicted to occur by elasticity theory when the size of an island becomes larger than the stress domain size $[9,10,20]$. The question now is whether the ratio of critical droplet diameter to stripe width agrees with theory. 


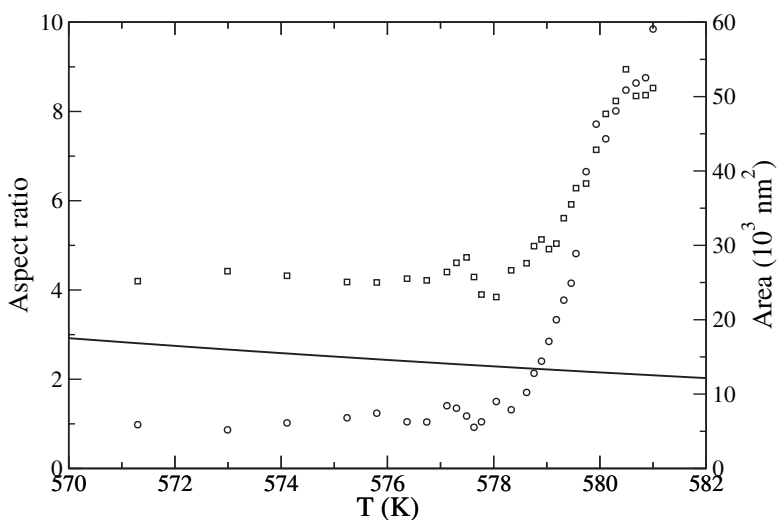

FIG. 3. Aspect ratio (circles) as a function of temperature showing a droplet undergoing a shape transition. The area of the droplet (squares) can be seen to increase dramatically as $\mathrm{Pb}$ is transported away from the droplet. The solid line indicates the equilibrium area of a circular droplet deduced from Refs. [18,19].

Measuring the critical ratio from Fig. 3 is complicated by the fact that besides the shape of the droplet changing with temperature, the area of the droplet is also changing (as seen in the figure). The reason for this change can be understood by considering the local environment of the islands shown in Fig. 1(a). As a function of temperature the relative fractions of the total surface area that is occupied by each of the two surface phases has to be approximately conserved [23]. As shown in Fig. 1(a), surrounding each terrace there is a step decorated with a stripe of alloy phase, which is close to its equilibrium width. As the temperature is lowered, $\mathrm{Pb}$ migrates from the antidroplets to the bounding stripes in order to allow the stripe width to shrink. This results in an increase in the area of the antidroplets with increasing temperature. This effect accounts for the droplet area changes seen in Figs. 1(b)-1(1). To determine accurately the critical ratio of droplet size to stripe width, this area change must be taken into account. To do this we plot the length of the short and the long axes of the antidroplet as a function of island area, rather than temperature, as shown in Fig. 4. Again, it is clear that there is a sharp transition at a well-defined droplet area [24]. To estimate this droplet area, we have determined the largest island area where the long and the short axis are no longer the same with their uncertainty. This uncertainty was estimated as $\pm 25 \mathrm{~nm}$ by measuring the fluctuation in each axis near the bifurcation point. Thus, combining Figs. 3 and 4 both the size and temperature of a critical droplet is now known. We have made this measurement for several droplets of different critical areas (and temperatures). The result of this analysis is shown in Fig. 5, which shows the critical droplet size as a function of stripe width. Although there is some scatter, the critical size is roughly proportional to the equilibrium stripe width. By performing a linear leastsquares fit we estimate that the value of the ratio of the

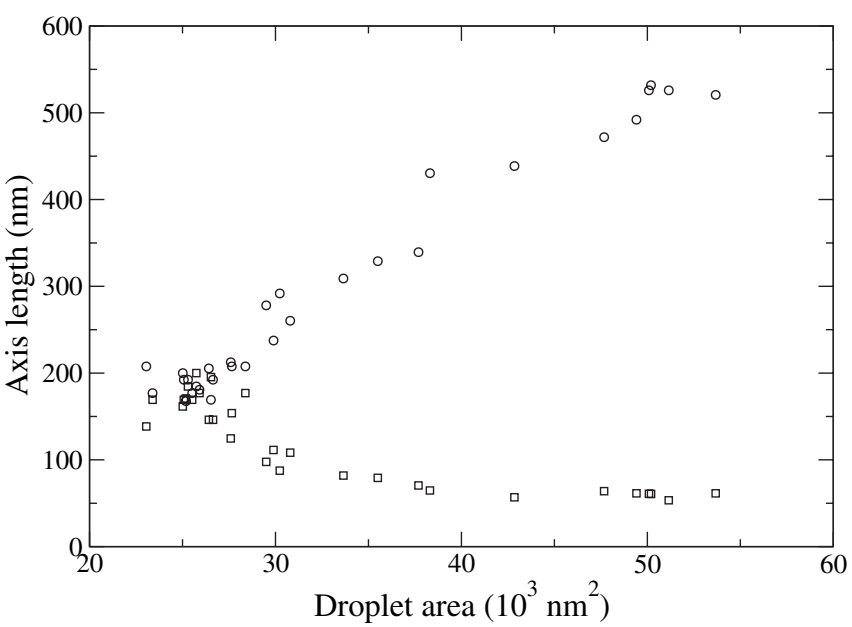

FIG. 4. Dimensions of a stripe showing the lengths of the long (circles) and short (squares) axes of an ellipse that was fitted to the shape of the stripe as it goes back through the shape transition. On the left-hand side of the graph, the shape of the droplet is circular again. For circular droplets, the difference between the short and long axis on the left-hand side of the graph is the result of thermal fluctuations that are picked up by the automated analysis routine. In this case the (reverse) shape transition took place at an area of $28 \pm 2 \times 10^{3} \mathrm{~nm}^{2}$ and a temperature of $580 \mathrm{~K}$.

critical droplet diameter to equilibrium width is $1.5 \pm 0.1$. This measurement is in reasonable agreement with the value of approximately 1.64 we predicted above for stress domains, given the simplifying assumptions of our calculation [25] and the experimental uncertainties.

Another aspect of the shape transition predicted by elasticity theory is that the equilibrium shape of the elongated islands should be a dog-bone-like shape with con-

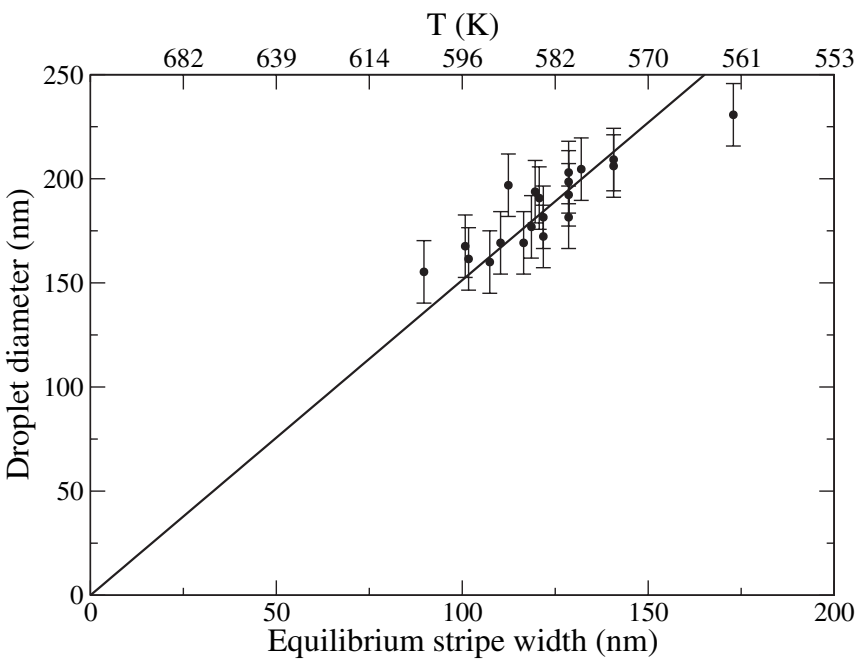

FIG. 5. Droplet diameter vs equilibrium stripe width at the shape transition temperature. The equilibrium stripe widths were determined from previous measurements, Refs. [18,19]. 
cave sides [8]. A careful examination of Fig. 1 reveals that the shape of a droplet as it goes through the transition does indeed follow the shapes predicted by the calculations. In the latter stages of the transition, where the length of the stripe is large with respect to its width, we indeed find the dog-bone shape, as can be seen in Figs. 1(d)-1(1). This aspect of the shape transition further supports the interpretation of the shape transition in terms of stress domains.

We have presented measurements illustrating a reversible shape transition of $\mathrm{Pb}$ overlayer islands in the $\mathrm{Pb} / \mathrm{Cu}(111)$ system. Isolated droplets assume an elongated shape when temperature is raised. Because of the reversibility of the transition we are confident that it is thermodynamic in origin. The fact that the details of the shape transition are so close to those predicted by stress domain theory supports our previous conclusions about the importance of elastic relaxations in the $\mathrm{Pb} / \mathrm{Cu}(111)$ system [1719]. It is striking that long-range elastic interactions, which are weak on an atom-by-atom basis, can determine the morphology of islands on length scales of hundreds of nanometers.

We gratefully acknowledge fruitful discussions with F. Léonard and J. B. Hannon. This work was supported by the U.S. Department of Energy, Office of Basic Energy Sciences, Division of Materials Science and Engineering. Sandia is a multiprogram laboratory operated by Sandia Corporation, a Lockheed Martin Company, for the United States Department of Energy's National Nuclear Security Administration under Contract No. DE-AC04-94AL85000.

[1] A. Piminelli and J. Villain, in Physics of Crystal Growth (Cambridge University Press, Cambridge, England, 1998), p. 43.

[2] V. I. Marchenko, JETP Lett. 33, 381 (1981).

[3] V. I. Marchenko and A. Ya. Parshin, Sov. Phys. JETP 52, 129 (1980).

[4] M. M. Hurley and S. J. Singer, Phys. Rev. B 46, R5783 (1992).

[5] O.L. Alerhand, D. Vanderbilt, R. D. Meade, and J.D. Joannopoulos, Phys. Rev. Lett. 61, 1973 (1988).
[6] K.-O. Ng and D. Vanderbilt, Phys. Rev. B 52, 2177 (1995).

[7] V. Zielasek, F. Liu, Y. Zhao, J. B. Maxson, and M. G. Lagally, Phys. Rev. B 64, 201320(R) (2001).

[8] G. E. Thayer, J. B. Hannon, and R. M. Tromp, Nat. Mater. 3, 95 (2004).

[9] J. Tersoff and R. M. Tromp, Phys. Rev. Lett. 70, 2782 (1993).

[10] A. Li, F. Liu, and M. G. Lagally, Phys. Rev. Lett. 85, 1922 (2000).

[11] E. Bauer, Rep. Prog. Phys. 57, 895 (1994).

[12] J. Henrion and G. E. Rhead, Surf. Sci. 29, 20 (1972).

[13] G. Meyer, M. Michailov, and M. Henzler, Surf. Sci. 202, 125 (1988).

[14] B. H. Müller, Th. Schmidt, and M. Henzler, Surf. Sci. 376, 123 (1997).

[15] C. Nagl, O. Haller, E. Platzgummer, M. Schmid, and P. Varga, Surf. Sci. 321, 237 (1994).

[16] Y. S. Chu, I. K. Robinson, and A. A. Gewirth, Phys. Rev. B 55, 7945 (1997).

[17] R. van Gastel, R. Plass, N. C. Bartelt, and G. L. Kellogg, Phys. Rev. Lett. 91, 055503 (2003).

[18] R. van Gastel, N. C. Bartelt, P. J. Feibelman, F. Léonard, and G. L. Kellogg, Phys. Rev. B 70, 245413 (2004).

[19] R. Plass, N. C. Bartelt, and G. L. Kellogg, J. Phys. Condens. Matter 14, 4227 (2002).

[20] H. M. McConnell, J. Phys. Chem. 94, 4728 (1990); J. M. Deutch and F.E. Low, J. Phys. Chem. 96, 7097 (1992).

[21] V. B. Shenoy, Appl. Phys. Lett. 85, 2376 (2004).

[22] F. Léonard, N. C. Bartelt, and G. L. Kellogg, Phys. Rev. B 71, 045416 (2005).

[23] The relative area of the two phases is not precisely conserved with increasing temperature because the $\mathrm{Pb}$ density of the two phases varies slightly with temperature. However, this effect is small over the narrow temperature range of these experiments.

[24] Above the transition, the width of the elongated droplet decreases with increasing droplet size. This effect is in part due to the fact that the larger island sizes correspond to larger temperatures and the equilibrium stripe width decreases with increasing temperature.

[25] For example, we have neglected the elastic anisotropy of the substrate. See Ref. [22] for a discussion of effects of this anisotropy. 